\title{
THE EFFECTS OF CO2 LASER ON ORAL LICHEN PLANUS AND LICHENOID LESIONS
}

T.C. Kok, S.T. Ong. The effects of CO2 laser on oral lichen planus and lichenoid lesions. Annal Dent Univ Malaya 2001; 8: 35-42.

\section{ABSTRACT}

The purpose of this study is to assess the effectiveness of $\mathrm{CO} 2$ laser in relieving symptoms associated with Oral lichen planus (OLP) and lichenoid lesions (OLL) and the event of healing. Six patients were selected for this study with 13 sites treated. Each lesion was ablated with CO2 laser and the wound allowed to heal undisturbed. Prior to treatment, 4 patients had both unprovoked and provoked pain and 2 patients rated for provoked pain only. After laser ablation, five out of six patients treated recorded no pain / 0 pain score. One month post-laser, the treated area was almost the same colour as the surrounding normal mucosa and soft in texture in all but one patient in which there was some fibrosis and residual plaque-like patch. We conclude that the use of $\mathrm{CO} 2$ laser in the treatment of OLP and OLL shows positive results in relieving symptoms associated with these lesions.

Key words: oral lichen planus, pain, treatment, $\mathrm{CO} 2$ laser, wound healing

\section{INTRODUCTION}

Lichen planus is an idiopathic chronic inflammatory disease that affects the skin and / or mucosa of squamous cell origin, characterised histologically by hyperorthokeratosis or hyperparakeratosis, degenerative changes to the basal cells and a band-like subepithelial infiltrate composed predominantly of lymphocytes. Oral lichenoid lesions (OLL) resemble oral lichen planus clinically and histologically and have been attributed to various drugs, foodstuff and dental materials. The WHO criteria for lichen planus do not differentiate between the two conditions, although it has been suggested that OLL has a more diffuse lymphocytic infiltrate containing eosinophils with perivascular infiltration (1).

Oral lichen planus (OLP) is a disease of adulthood with a mean age of onset in the fourth and fifth decade and a predilection for females. It may occur independent of skin lesions and has been reported to affect $0.1 \%$ to $2.2 \%$ of the population sampled $(2,3,4)$. In Malaysia, the reported prevalence of OLP is $0.38 \%$ of the population (5).

Oral lichen planus is commonly asymptomatic. In the absence of symptoms, treatment is generally not warranted since none of the available treatment is specific or universally successful and all can have adverse effects (6). There may be some clinical benefit in changing the medication if drug related lichen planus
Original Article

\begin{tabular}{l} 
T.C. Kok, S.T. Ong* \\
Dental Specialist \\
Oral Surgery Clinic \\
Hospital Melaka \\
*Associate Professor \\
Department of Oral and Maxillofacial Surgery \\
Faculty of Dentistry \\
University of Malaya \\
50603 Kuala Lumpur, Malaysia \\
Corresponding author - S.T. Ong \\
\hline
\end{tabular}

is suspected, although this is not unequivocally successful. Studies have shown that some lesions resembling OLP may occur in direct relationship to amalgam restorations. Some of these lesions may improve after substitution of the amalgam by other materials $(7,8,9)$. However, composite restorations have also been implicated in OLL (10) and so the wholesale replacement of amalgam is not warranted. Mechanical trauma or irritants from sharp fillings or rough margins should however be eliminated.

Drug therapy has been the mainstay of treatment for symptomatic cases with symptoms ranging from burning sensation on eating spicy food, soreness/discomfort to ulcer. Corticosteroids in topical application are usually the treatment of choice initially because it can be effectively delivered to the lesion surface with minimal side effects (11). Systemic steroids are indicated only rarely for recalcitrant lesions with severe signs and symptoms

Surgical excision of oral lichen planus has the advantage of eliminating the lesion and if submitted for histopathological examination would give a histopathological diagnosis. However it is not a common practice mainly because of the widespread nature of OLP on the oral mucosa. Carbon dioxide (CO2) laser offers an alternative to the scalpel, in the treatment of symptomatic lichen planus $(12,13)$. Laser, an acronym for light amplification by stimulated emission of radiation, is a system where energy can be harnessed and channelled to produce the desired effects, in this situation, as a surgical tool. A laser beam, being monochromic, coherent and collimated is highly precise and its specific wavelength determines its optical properties such as laser spot size, power and interaction at laser-tissue interface. The power and speed of laser output is also determined by the waveform, which may be modified and controlled within limits by the operator.

There are a number of lasers being used for surgery. These include CO2, Argon, Nd-YAG, Er-YAG, Ho-YAG etc. The choice of laser for a particular surgery depends 
on a few factors, namely precision and depth of tissue ablation, haemostasis, any preference for pigmented tissue and the effectiveness in a fluid filled environment such as the joint cavity. $\mathrm{CO} 2$ laser with a wavelength of $10.6 \mu \mathrm{m}$ is strongly absorbed by water. The absorbed energy causes vaporisation of the intra-cellular and extracellular fluid and destruction of the cell membranes. It is consistently absorbed within $0.5 \mathrm{~mm}$ of the tissue surface without regards for pigmentation and is therefore well suited for the treatment of superficial mucosal lesion.

The use of $\mathrm{CO} 2$ laser as a surgical tool first started in this centre in 1992 and has been used occasionally for ablation of OLP and OLL. However, there was no objective assessment of the outcome of treatment. The objective of this prospective study is to assess the effects of $\mathrm{CO} 2$ laser and its effectiveness as an alternative treatment for these lesions, as conventional drug therapy has not been universally successful. They are assessed in terms of relief of symptoms, morbidity of the treatment and clinical and histological changes if any.

\section{MATERIALS AND METHODS}

New patients with OLP or OLL, who registered with the Department of Oral \& Maxillofacial Surgery, were offered $\mathrm{CO} 2$ laser ablation of the lesions as the treatment of choice. To be included in the study, they should fulfil the following criteria:

a) Histopathological confirmation of lichen planus / lichenoid lesion

b) The patient must present with one or more symptoms related to the lesion

1. For this prospective study, the patients gave informed consent with respect to the $\mathrm{CO} 2$ laser treatment and for inclusion into the study.

2. A questionnaire is given to each patient before the laser treatment. The information required include socio-demographic and medical details, symptoms related to OLP and any previous and current therapy given. The patient was required to indicate on a Verbal Rating Score (VRS) and a $10 \mathrm{~cm}$ Visual Analogue Scale (VAS), the level of pain or discomfort experienced.
3. The lesions were charted by drawing on a standard diagram and by photographs. The procedure was repeated at 1 month and 3 months after laser therapy.

4. Treatment protocol:

(a) The OLP lesion was ablated under local anaesthesia with $\mathrm{CO} 2$ laser in continuous wave mode moving in a criss-cross pattern until the submucosa is reached. It this study, the machine used was Luxar LX-20 $0^{\oplus}$ at the setting of $10 \mathrm{~W}$ continuous mode. A margin of $3 \mathrm{~mm}$ around each lesion was included in the ablation. (The power output of each machine may vary. It should therefore be individually calibrated and adjusted to the optimal power setting.)

(b) The laser defect was allowed to heal undisturbed and the patient reviewed and assessed at intervals of 1 week, 2 weeks 1,3 , and 6 months.

5. Evaluation:

(a) At each post-operative review, the patient was again requested to score the level of pain / discomfort experienced on the Verbal Rating Score and the Visual Analogue Scale.

(b) Patients were evaluated on relief of symptoms by comparing the pre- and post-laser intensity of pain / discomfort.

(c) The effectiveness of removal of lesion was assessed clinically and histologically. Biopsy was taken 3 months after the procedure for histopathological examination and the results compared with the report of initial lesion.

(d) No statistical analysis was done in view of the small sample size.

\section{RESULTS}

During the period of the clinical study, there were five patients with Oral Lichen Planus (OLP) and one with Oral Lichenoid Lesion (OLL) who fulfilled the criteria and consented to $\mathrm{CO} 2$ Laser ablation of the lesions (Table 1).

Table 1: Details of patients in the clinical study

\begin{tabular}{clll}
\hline INDEX & AGE/GENDER/RACE & $\begin{array}{l}\text { HISTOLOGICAL DIAGNOSIS \& } \\
\text { CLINICAL FORM }\end{array}$ & CHIEF COMPLAINT \\
\hline 1 & $50 \mathrm{yr} /$ female / Indian & Lichen planus, Reticular & Burning sensation on eating spicy food \\
2 & $65 \mathrm{yr} /$ female / Chinese & Lichen planus, Reticular & Soreness/ discomfort \\
3 & $60 \mathrm{yr} /$ female / Chinese & Lichen planus, Reticular & Burning sensation on eating spicy food \\
4 & $41 \mathrm{yr} /$ male / Indian & Lichen planus, Reticular & Soreness /discomfort \\
5 & $49 \mathrm{yr} /$ male / Chinese & Lichen planus, Plaque-like & Soreness / discomfort \\
6 & $65 \mathrm{yr} /$ male / Indian & Lichenoid reaction, Reticular & Burning sensation on eating spicy food \\
\hline
\end{tabular}


Table 2: Pain Score on Verbal Rating Score and Visual Analogue Scale

\begin{tabular}{|c|c|c|c|c|c|}
\hline \multirow[t]{2}{*}{ Index } & \multirow[t]{2}{*}{ SITES } & \multicolumn{2}{|c|}{ Before treatment } & \multicolumn{2}{|c|}{ After treatment } \\
\hline & & $\begin{array}{l}\text { Unprovoked } \\
\text { VRS \& VAS }\end{array}$ & $\begin{array}{l}\text { Provoked } \\
\text { VRS \& VAS }\end{array}$ & $\begin{array}{l}\text { Unprovoked } \\
\text { VRS \& VAS }\end{array}$ & $\begin{array}{l}\text { Provoked } \\
\text { VRS \& VAS }\end{array}$ \\
\hline 1 & $\begin{array}{l}\text { Left } B M \\
\text { Left lateral tongue }\end{array}$ & No pain $(0)$ & Mild pain (4) & No pain $(0)$ & No pain $(0)$ \\
\hline 2 & $\begin{array}{l}\text { Right BM } \\
\text { Left BM } \\
\text { Lower left alveolus }\end{array}$ & Mild pain (2) & Mild pain (4) & No pain $(0)$ & No pain $(0)$ \\
\hline 3 & $\begin{array}{l}\text { Left BM } \\
\text { Left lateral tongue }\end{array}$ & Mild pain (2) & Moderate pain (8) & No pain $(0)$ & No pain (0) \\
\hline 4 & $\begin{array}{l}\text { Right BM } \\
\text { Left BM } \\
\text { Right lateral tongue }\end{array}$ & Mild pain (4) & Moderate pain (7) & No pain (0) & No pain (0) \\
\hline 5 & Right BM & Moderate pain (8) & Moderate pain (8) & Moderate pain (8) & Moderate pain ( 8 ) \\
\hline 6 & $\begin{array}{l}\text { Right BM } \\
\text { Left BM }\end{array}$ & No pain $(0)$ & Mild pain (2) & No pain $(0)$ & No pain $(0)$ \\
\hline
\end{tabular}

$\mathrm{BM}=$ Buccal Mucosa

Of the six patients in the study, three were males and three were females. The age range was $41-65$ years (mean age of 55 years). The chief complaint was burning sensation (3 cases), and soreness/discomfort (3 cases). The majority of the cases ( 4 patients) presented with symptoms of less than 6 months' duration. All patients had involvement of the buccal mucosa either singly, bilateral or with additional involvement of another site. Five patients had a past medical history ranging from allergies ( 2 cases), hypertension (1 case), diabetes mellitus (1 case) and cancer of the thyroid gland (1 case). Three patients were on medication for their medical condition and two patients had a smoking habit. A total of thirteen sites were treated. The follow-up period for these six patients in the clinical study was 3 - 10 months.

\section{Pain Scores}

Before treatment, all patients reported varying degrees of oral discomfort which were assessed with a Verbal Rating Score and a Visual Analogue Scale, divided into unprovoked persistent soreness/discomfort and provoked pain / discomfort (Table 2).

After treatment, 5 patients rated no pain. The rating on VAS for these patients was 0 pain scores for both unprovoked and provoked pain. One patient (case No. 5) reported no improvement.

\section{Clinical course of laser wound healing}

The appearance of an initial lesion is as shown in Figure 1. During laser ablation, superficial layer of the mucosa was removed. There was minimal or no bleeding and a yellowish-brown carbonised layer formed on the lased surface. At one week review, all patients reported pain that started on the third post-laser day. Majority (4 patients) had mild pain while one patient had moderate pain; controlled with mild analgesics. The pain for these patients lasted 2 - 3 days. One patient had severe pain that lasted more than 2 weeks. This was the patient who had the plaque-like form of lichen planus.

One week post-laser, the lased areas were covered with yellowish-white fibrinous coagulum (Figure 2). The original outlines of the lased areas were still visible. There is a distinct boundary between the treated area and the adjacent epithelium but no swelling or erythema (inflammation) of the surrouding tissue.

Two weeks post-laser, the fibrinous coagulum is still present with new epithelium proliferating at the margins. One month post-laser the healing of the lased areas was complete. The laser-treated mucosa was almost the same colour as the surrounding normal mucosa (Figure 3). The healed mucosa was soft in texture. However for the plaque-like form, there was some fibrosis and the plaque-like patch was still present but it was reduced in size.

\section{Histological appearance}

Histological examination on all six patients before laser treatment reported five cases with lichen planus (Figure 4) and one case with lichenoid reaction. Three months post-laser, one patient refused biopsy and another one was lost to follow-up. Biopsy was done on the remaining four patients. Three cases still showed the histological appearance of lichen planus/ lichenoid reaction as before the laser treatment, with one of them showing features of mild dysplasia (drop-shaped rete processes, loss of stratification in the basal cell layers and occasional hyperchromatic nuclei.) Only one case showed absence of evidence of lichen planus with the diagnosis of non-specific keratosis (Figure 5). In this case there was only a mild chronic inflammatory cell infiltrate with no basal cell liquefactive degeneration and lymphocytic band. 


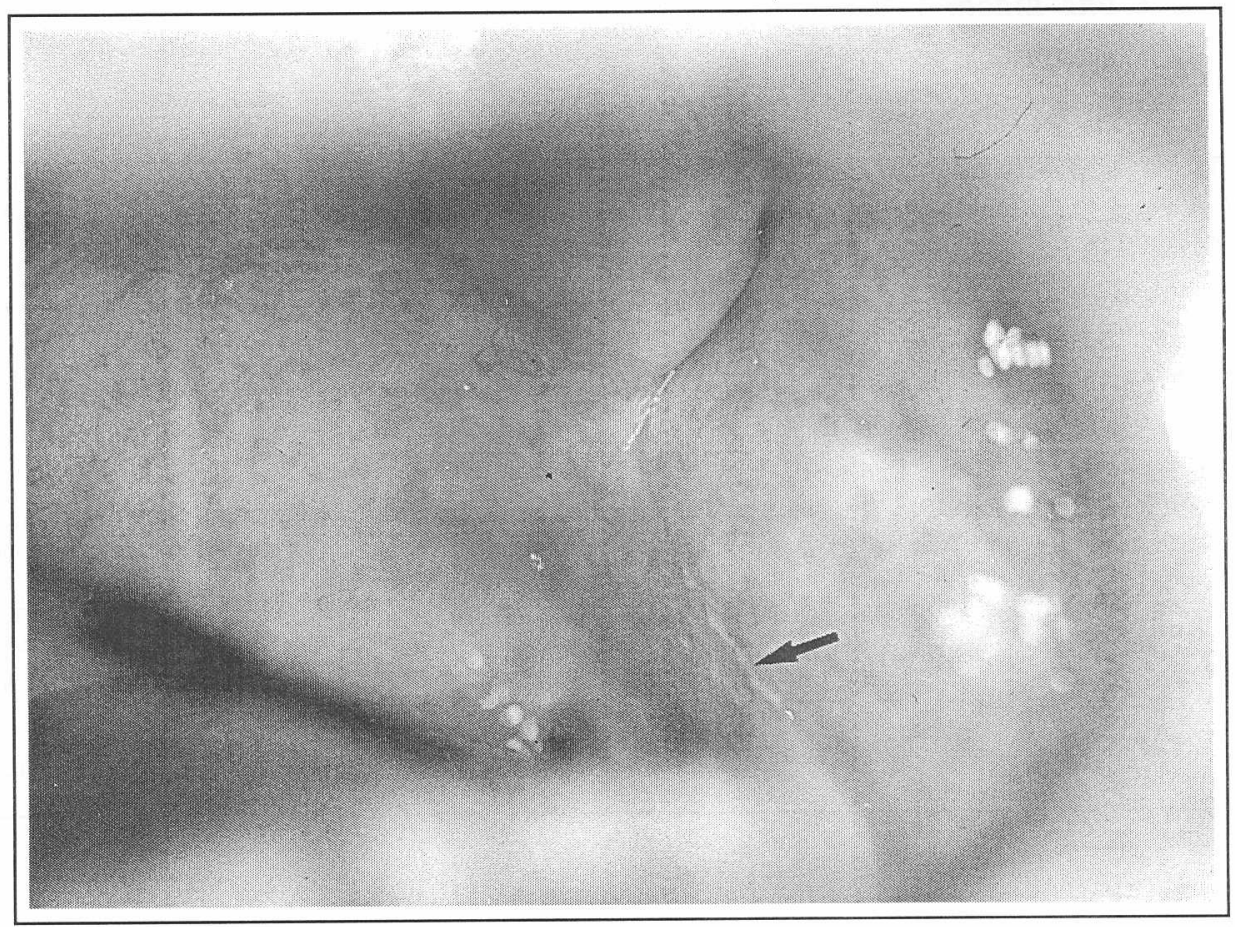

Figure 1. Lichen planus on the lower alveolar ridge of left retromolar area (arrow).

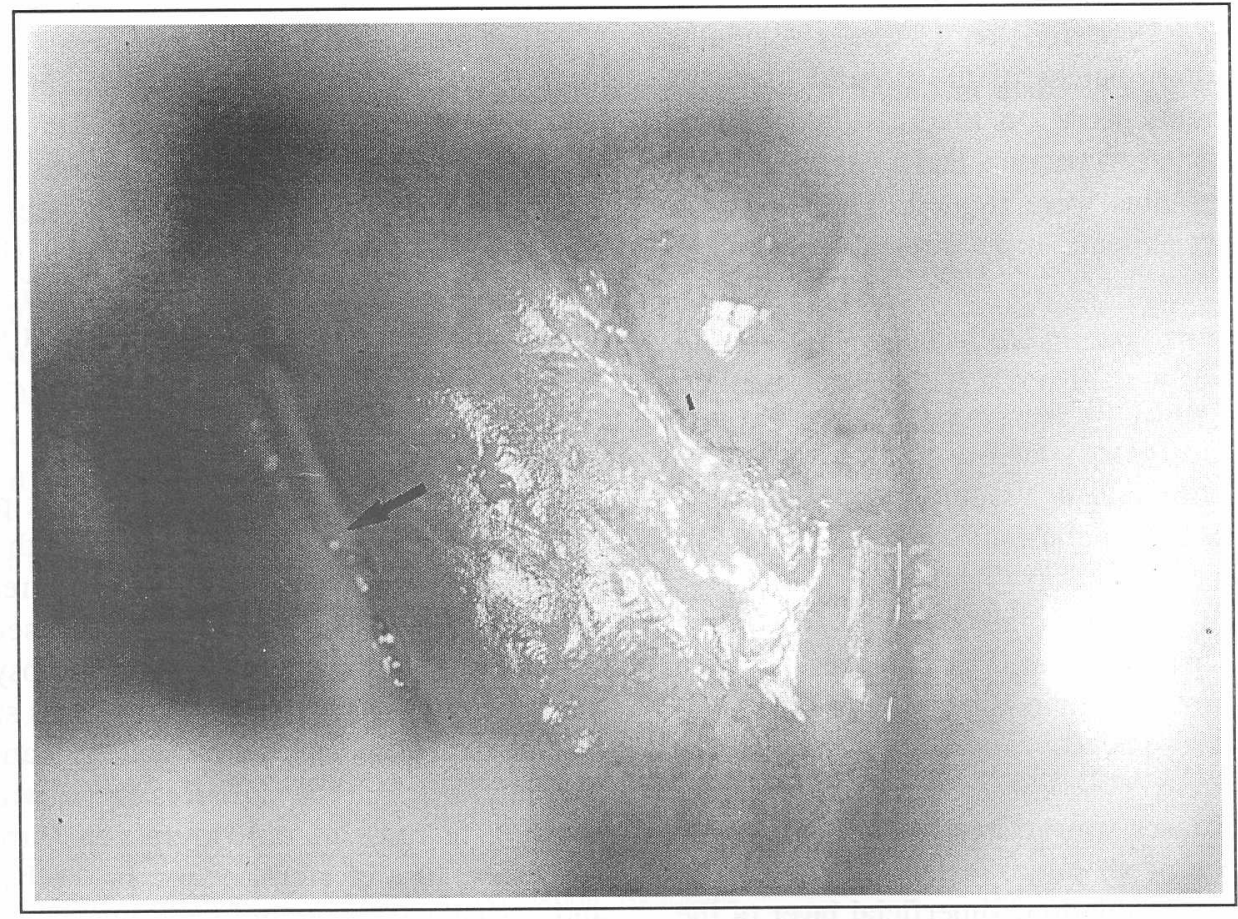

Figure 2: Laser wound at one-week review. There was a distinct boundary between the treated area and the adjacent mucosa. The laser area was covered with yellowish-white fibrinous coagulum (arrow). 


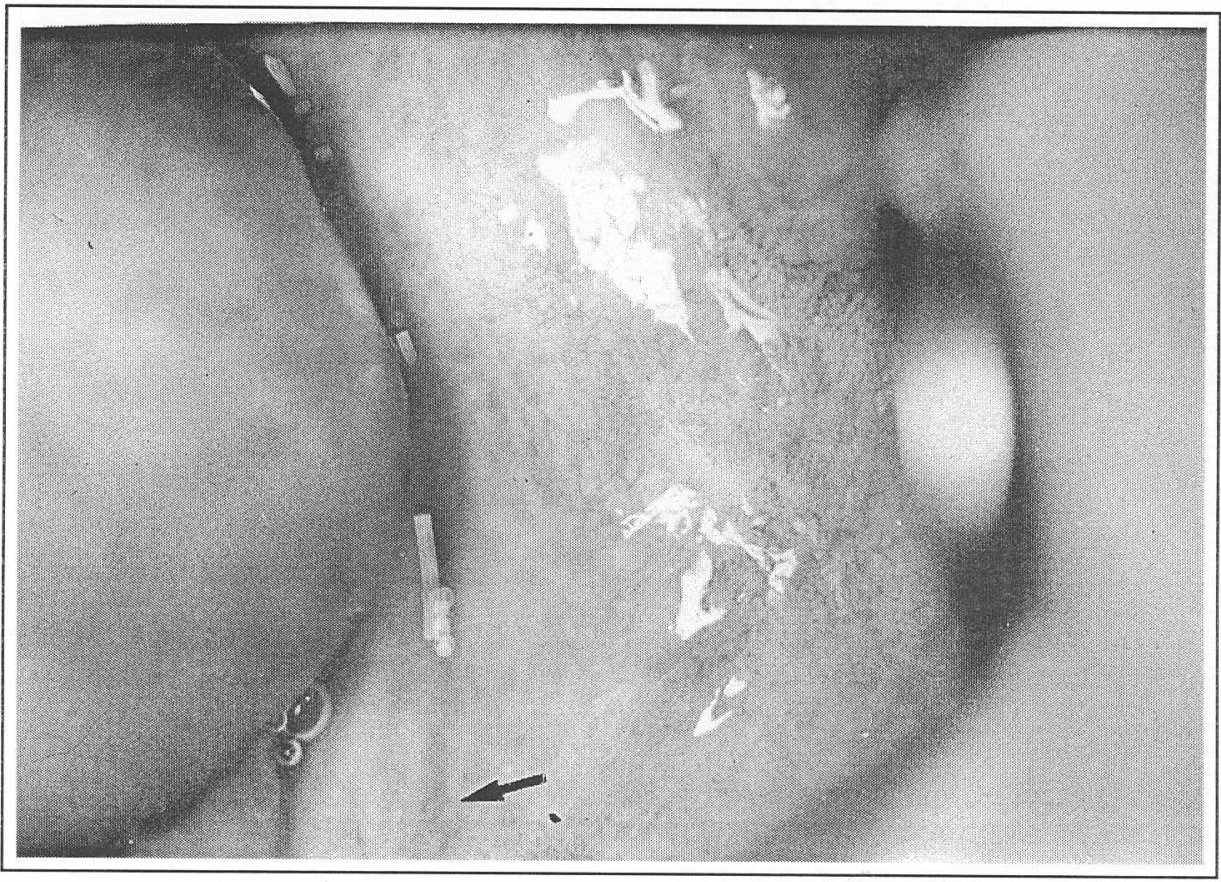

Figure 3: Area of previous OLP one month after laser ablation, showing good healing indistinguishable from adjacent mucosa (arrow).

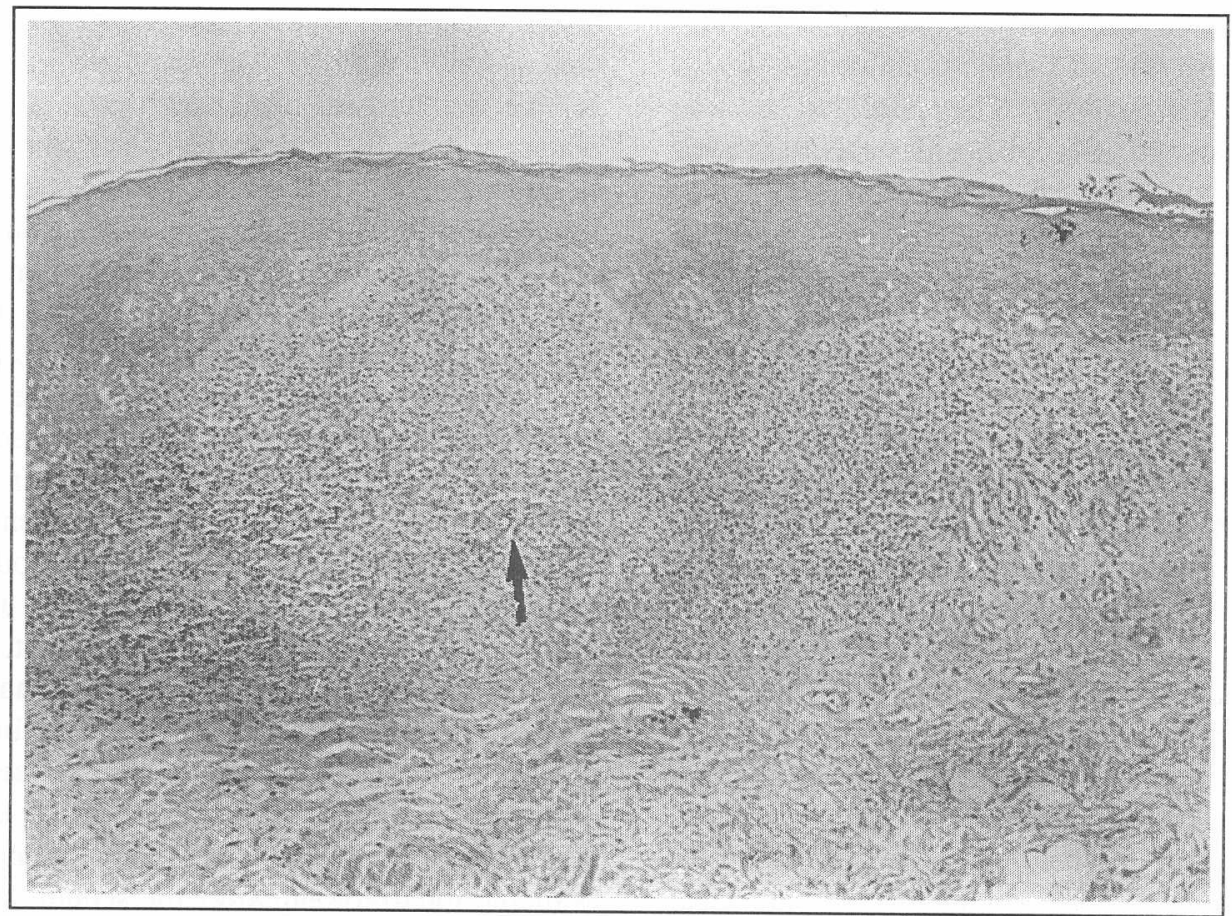

Figure 4: Histological appearance of OLP before laser ablation; the band of subepithelial lymphocytic inflitrate is evident (arrow). HPE X 40 


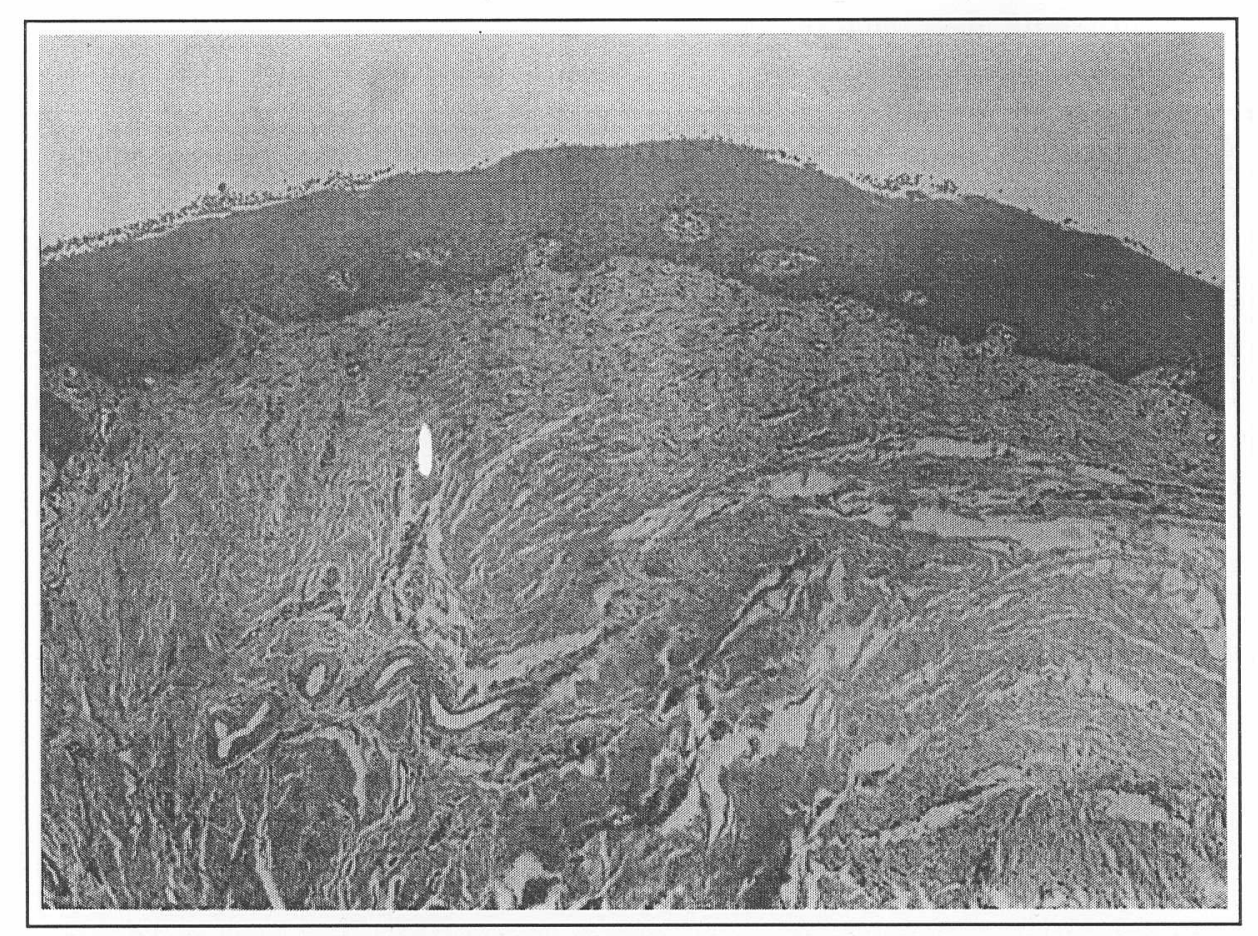

Figure 5: Histological appearance of the same site 3 months post-laser; showing keratosis and absence of subepithelial lymphocytic band. HPE X 40

\section{Recurrence}

In the follow-up period of 3 - 10 months, five of the patients did not show recurrence. One patient with the plaque-like form of lichen planus did not return for follow-up after 3 months. In this patient, the plaque-like area at the right buccal mucosa that was reduced in size in the first visit, was lased a second time but the patient did not return for follow-up. The case that showed histological appearance of mild dysplasia still showed clinically normal mucosa at 6 months follow up. The symptomatic improvement continued throughout the follow-up period.

\section{DISCUSSION}

The first component of disease management is patient education (11). The patient should be made aware of the nature of the disease, the unpredictable clinical course and the rationale of current therapeutic recommendations. The patient's failure to understand the aims of treatment often results in unnecessary disease exacerbation and a failure of the practitioner to meet the patient's expectations. The patient must be advised that lichen planus is characterised by unpredictable exacerbation that may in some cases require treatment for many years. It is also important to eliminate local or systemic factors that may be aggravating or precipitating oral lichen planus, for example drug induced lichenoid lesions, possible electrogalvanisme or haematinic deficiency. For lesions showing dysplasia, the ideal treatment is complete excision with regular followup. However, a complete elimination of dysplasia may be made difficult by wide field change.
$\mathrm{CO} 2$ laser is being increasingly used in soft tissue surgery including the removal of premalignant lesions. Surgical ablation with $\mathrm{CO} 2$ laser avoids the systemic adverse effects of drug therapy in the treatment of lichen planus. In addition, it offers the advantages of minimal damage to surrounding healthy tissues and excellent wound healing with little scarring. It also appears to relieve the burning sensation associated with lichen planus and enables the patient to tolerate spicy food. A study by Loh (14) on ten OLP patients treated with $\mathrm{CO} 2$ laser reported that all the patients had immediate relief of associated burning sensation and tolerance to hot and spicy food. This improvement lasted throughout the follow-up period of 6 months to 4 years. Five out of the six patients in this study reported significant improvement in their symptoms after laser treatment as documented in their verbal rating score and visual analogue scale. They no longer had soreness/discomfort or burning sensation on eating spicy food. In our study so far, with a follow-up period of 3-10 months, the symptomatic improvement is still present. The only case that had no symptomatic improvement had an extensive plaque-like type of OLP on the right buccal mucosa, compared to the other cases that presented with the reticular form. This patient was given laser treatment twice due to the extensive involvement. He was not available for follow-up after the second laser treatment. There was no symptomatic improvement after the first treatment as the lesion was still present although reduced in size. Therefore it is difficult to draw any firm conclusions from this patient.

There was clinical elimination of the lesions in five of the patients after laser treatment. The lased area was almost imperceptible compared to the adjacent normal 
mucosa. Loh (14) in his study also found the healed lased mucosa to be almost similar to the surrounding normal mucosa. Fisher and Frame (15) found laser wounds to heal well with little scarring. However in this study there was one case with some fibrosis after laser treatment. This was the case with extensive plaque-like involvement. The extensive area lased may have contributed to this. The depth and width of affected surrounding normal tissues contributes to the final condition of the wound (14).

So far in the follow-up of the patients for 3-10 months there has not been any clinical recurrence but a longer follow-up is necessary. Loh (14) in a follow-up period of up to 4 years found $80 \%$ clinical elimination of the condition with $20 \%$ requiring subsequent laser therapy. He found that when lichen planus recurred, it did not occur in the lased area, but in new untreated area. This could not be verified in the present study.

Biological and clinical studies have shown that wounds produced by the $\mathrm{CO} 2$ laser behave in a different manner than those produced by the scalpel (15). Healing after laser surgery differs from that of classical healing by secondary intention. Such a wound is not a burn but rather there is an almost instantaneous vaporisation of intracellular fluid with disintegration of the cell structure (16). The majority of patients experienced minimal to moderate post-operative pain, which started only after 3 days. This is due to the initial lack of inflammation with laser wounds (15). The fibrinous coagulum covering the laser wound until it completely epithelialises also acts as an impermeable dressing.

There was no histological improvement after laser treatment except for one case. This case showed nonspecific keratosis with no evidence of OLP. This finding in only one case raises the question of whether the biopsy was taken at the wrong site, as the healed lased area looked similar to the adjacent normal mucosa. However Loh (14) in his study, did find histological changes post laser, with a reduction in the sub-basal lymphocytic band and the degree of acanthosis. The significance of this finding is however unclear.

It was reported that mild to moderate dysplasia occurs in $25 \%$ of OLP (17) and it is considered to fulfil the WHO criteria for a premalignant lesion (18). There was one case that showed mild dysplasia after laser treatment. Such dysplasia was not evident in the prelaser biopsy because it may not be representative of the whole lesion. The slight potential for malignant transformation of OLP is most probably intrinsic in the tissue and not provoked by $\mathrm{CO} 2$ laser treatment. The use of $\mathrm{CO} 2$ laser in the treatment of pre-malignant lesions like leukoplakia is well documented and there had been no reported cases of malignant transformation directly attributed to the effects of $\mathrm{CO} 2$ laser. Apfelberg et al (19) showed in an experimental study on mouse fibroblasts that $\mathrm{CO} 2$ laser does not induce malignant transformation. Fisher and Frame (15) speculated that shedding of possibly malignant cells or immunologically active cell particles is unlikely during laser surgery, because cells coming into contact with the laser beam are destroyed and the temperature is sufficiently high to denature cell protein. Roodenburg et al (20) excised 103 leukoplakic lesions with CO2 laser, of which 33 had dysplasia ranging from mild to severe. They achieved a cure rate of $90 \%$ with a mean follow-up period of 5.3 years and none of these lesions transformed into squamous cell carcinoma.

The mechanism by which laser brings about the symptomatic and clinical improvement in OLP is unknown. As lichen planus is a systemic disease, it is possible that laser surgery has caused a systemic change through its local action on the oral tissues (14). The immunological status is perhaps altered. Oka et al (21) treated eight cases of OLP with CO2 laser and claimed that $\operatorname{IgG}, \operatorname{Ig} \mathrm{A}$ and $\operatorname{IgM}$ levels were unchanged before and after laser treatment. Other studies $(22,23)$ showed changes in IgG levels. Obviously there is a need for further studies particularly on the serum levels of immunoglobulins and complements to see if an immune response has been altered which can relate to the improvement of the condition.

The present study is limited in the number of patients and length of follow-up and could at best be considered a preliminary study. Nonetheless, it does show the fact that $\mathrm{CO} 2$ laser ablation of OLP causes minimal morbidity and there is also satisfactory healing and control of discomfort / pain associated with this lesion. It could therefore be an effective treatment modality for OLP. However, a regular and longer followup is necessary to assess the lesion and possible longterm side effects of this form of treatment. Dysplastic changes if detected and adequately excised at its early stages, may help reduce the occurrence of malignant transformation. In addition, further study on clinical and immunological aspects should continue to expand on the existing information. It is necessary to take note that all surgical lasers come into Class IV category posing significant risks to unprotected eye and skin and also a fire hazard. Therefore laser safety precautions should be taken.

\section{ACKNOWLEDGEMENT}

The authors would like to thank the Oral Pathologists of Faculty of Dentistry, University of Malaya for reporting on the biopsy specimen and Professor Dr. Siar Chong Huat for providing the prints of histological slides. This project was partially funded by vote $F$ of University of Malaya and the data forms part of the thesis in fulfillment of Master of Dental Science, University of Malaya of the first author.

\section{REFERENCES}

1. Van den Haute, Antoine JL, Lachapelle JM. Histopathological discriminant criteria between lichenoid drug eruptions and idiopathic lichen planus: retrospective study on selected samples. Dermatologica 1989; 179: 10-13. 
2. Pindborg JJ, Mehta FS, Daftary DK et al. Prevalence of oral lichen planus among 7639 Indian villagers in Kerala, South India. Acta Derm Venereol (Stockh) 1972; 52: 216-220.

3. Bouquot JE, Gorlin RJ. Leukoplakia, lichen planus and other keratoses in 23,616 white Americans over the age of 35 years. Oral Surg Oral Med Oral Pathol 1986; 61: 373-381.

4. Axell T, Rundquist L. Oral Licher planus - a demographic study. Community Dent Oral Epidemiol 1987; 15: 52-56.

5. Zain RB, Ikeda N, Razak IA et al. A national epidemiological survey of oral mucosal lesions in Malaysia. Com Dent Oral Epidemiol 1997; 25: 377383.

6. Scully $\mathrm{C}$ et al. Update on oral lichen planus: Aetiopathogenesis and management. Crit Rev Oral Biol Med 1998; 9: 86-122.

7. Bolewska J, Holmstrup P, Moller-Madsen B, Kenrad B, Danscher G. Amalgam associated mercury accumulations in normal oral mucosa, oral mucosal lesions of lichen planus and contact lesions associated with amalgam. J Oral Pathol Med 1990; 19: 39-42.

8. Ibbetson SH, Speight EL, Macleod RI et al. The relevance and effect of amalgam replacement in subjects with oral lichenoid reactions. Br J Dermatol 1996; 134: 420-423.

9. Smart ER, Macleod RI, Lawrence CM. Resolution of lichen planus following removal of amalgam restorations in patients with proven allergy to mercury salts: a pilot study. Br Dent J 1995; 178: 108-112.

10. Lind P. Oral lichenoid reactions related to composite restorations. Acta Odontol Scand 1988; 46: 63-65.

11. Vincent SD, Fotos PG, Baker KA, Williams TP. Oral lichen planus: the clinical, histological and therapeutic features of 100 cases. Oral Surg Oral Med Oral Pathol 1990; 70: 165-171.

12. Frame JW, Das Gupta AR, Dalton GA, Rhys-Evans $\mathrm{PH}$. Use of the $\mathrm{CO} 2$ laser in the management of premalignant lesions of the oral mucosa. J Laryngol Otol 1984; 98: 1251-1260.
13. Horch H, Gerlach KL, Schaefer HE. C02 laser surgery of oral premalignant lesions. Oral Maxillofac Surg 1986; 15: 19-24.

14. Loh HS. A clinical investigation of the management of oral lichen planus with $\mathrm{CO} 2$ laser surgery. J Clinical Med \& Surg 1992; 10: 445-449.

15. Fisher SE, Frame JW. The effects of the $\mathrm{CO} 2$ surgical laser in oral tissues. Br J Oral \& Maxillofacial Surg 1984; 22: 414-425.

16. Hall RR. The healing of tissues incised by a $\mathrm{CO} 2$ laser. Br J of Surg 1971; 58: 222.

17. De Jong WF, Albrecht M, Banoczy, van Der Waal. Epithelial dysplasia in oral lichen planus. A preliminary report of a Dutch-Hungarian study of 100 cases. Int J Oral Surg 1984; 13: 221-225.

18. Holmstrup P, Thorn JJ, Rindrum J, Pindborg JJ. Malignant development of lichen planus affected oral mucosa. J Oral Pathol 1988; 17: 219-225.

19. Apfelberg DB, Mittelman H, Chadi B, Maser M, Lash $\mathrm{H}$. Investigation of carcinogenic effects of in vitro argon and $\mathrm{C} 02$ laser exposure of fibroblasts. Laser Surg Med 1984; 4: 173-179.

20. Roodenberg JLN, Panders AK, Vermey MD. Carbon dioxide laser surgery of oral leukoplakia. Oral Surg Oral Med Oral Pathol 1991; 71: 670-674.

21. Oka M, Santoh E, Harada T, Yoshimura Y. Application of $\mathrm{CO} 2$ laser to oral lesions and immunological study of oral lichen planus in: Lasers in Dentistry. Yamamoto H, Atsumi K, Kusakari H (eds). Amsterdam. Elsevier Science Publishers 1989; 193-198.

22. Sklavounou AD, Laskaris G, Angelopoulos AP. Serum immunoglobulins and complement in oral lichen planus. Oral Surg Oral Med Oral Pathol 1983; 55: 47-51.

23. Lundstrom I. Serum immunoglobulins and autoantibodies in patients with oral lichen planus. Int J Oral Surg 1985; 14: 259-268. 